\title{
Freqüência cardíaca em homens imersos em diferentes temperaturas de água
}

\author{
Fabiane Graef \\ Leonardo Tartaruga \\ Cristine Alberton \\ Luiz Kruel
}

https://doi.org/10.5628/rpcd.05.03.266

\section{RESUMO}

O objetivo deste estudo foi analisar o comportamento da freqüência cardíaca de indivíduos imersos na posição vertical, na profundidade entre apêndice xifóide e ombros, em três temperaturas de água $\left(27,30\right.$ e $\left.33^{\circ} \mathrm{C}\right)$. A amostra foi composta por 14 indivíduos do sexo masculino, praticantes de atividades aquáticas, na faixa etária de 18 a 35 anos. A frequiência cardíaca foi verificada através de sensores de batimentos cardíacos da marca Polar, modelo Beat. O comportamento da freqüência cardíaca foi determinado através das variações existentes entre a freqüência cardíaca dos indivíduos na posição vertical fora de água e durante a imersão, em repouso. A análise dos dados foi feita utilizando-se estatística descritiva, análise de variância (ANO VA), com teste post-hoc de Bonferroni $(\mathrm{p} \leq 0,05)$. Verificou-se que houve bradicardia durante a imersão, nas três temperaturas aquáticas. A bradicardia média na temperatura de $33^{\circ} \mathrm{C}$ foi $17,85 \pm 10,67$ bpm; em $30^{\circ} \mathrm{C}$, foi $24,14 \pm 11,16 \mathrm{bpm}$; em $27^{\circ} \mathrm{C}$, foi

$33,75 \pm 11,27$ bpm. As diferenças entre as médias mostraram-se estatisticamente significativas somente entre a temperatura de $27^{\circ} \mathrm{C}$ e a temperatura de $33^{\circ} \mathrm{C}$. Conclui-se que, durante a imersão aquática em temperaturas variando entre 27 e $33^{\circ} \mathrm{C}$, a bradicardia tende a aumentar com a diminuição da temperatura.

Palavras-chave: freqüência cardíaca, imersão, temperatura aquática.
Universidade Federal do Rio Grande do Sul Escola Superior de Educação Física Porto Alegre, Brasil

\author{
ABSTRACT \\ Heart rate frequency in immersed individuals over different \\ water temperatures
}

Purpose of this study was to analyze the heart rate behavior of immersed individuals in the vertical position, at a depth between the xiphoid process and the shoulders, in three water temperatures $(27,30$ and $33^{\circ} \mathrm{C}$ ). Sample was composed of 14 male subjects aged 18 to 35 , who practice water activities. Heart rate was monitored with Polar heart beat sensors, Beat model. The heart rate behavior was determined through the existing variations between the individual's heart rate in the vertical position outside the water and during immersion, at rest. The analysis of the data was conducted using descriptive statistics, variance analysis (ANOVA), with Bonferroni's post-hoc test $(p \leq 0,05)$. Bradycardia was observed during immersion in all three water temperatures. The average bradycardia at $33^{\circ} \mathrm{C}$ was $17,85 \pm 10,67$ beat. $\mathrm{min}^{-1}$; at $30^{\circ} \mathrm{C}$, it was $24,14 \pm 11,16$ beat. $\mathrm{min}^{-1}$; at $27^{\circ} \mathrm{C}$, it was $33,75 \pm 11,27$ beat. $\mathrm{min}^{-1}$. The differences between the averages were only statistically significant between $27^{\circ} \mathrm{C}$ and $33^{\circ} \mathrm{C}$. Therefore, during water immersion in temperatures varying between 27 and $33^{\circ} \mathrm{C}$, bradycardia tends to increase with the reduction of water temperature.

Key Words: heart rate, immersion, water temperature. 


\section{INTRODUÇÃO}

O número de praticantes de atividades físicas aquáticas vem tornando -se cada vez mais expressivo, incluindo tanto indivíduos saudáveis quanto pessoas com necessidades especiais (21). Embora os prazeres e benefícios do trabalho aquático tenham sido descobertos há vários séculos, somente nos últimos anos mostraram-se amplamente divulgados e popularizados.

Sabe-se que o sistema cardiovascular reage de formas diferenciadas em situação de imersão, de acordo com a posição adotada, com a ausência ou presença de esforço (e sua intensidade), com o tipo de exercício, com a profundidade da imersão e com a temperatura da água (19). O exercício físico realizado em meio aquático produz respostas fisiológicas diferentes daquelas relatadas fora deste ambiente, devido ao efeito hidrostático no sistema cardiovascular e à intensificação da perda de calor na água (2); mesmo em situação de repouso, existem modificações cardiovasculares determinadas pela imersão na água (9). Dentre os parâmetros circulatórios influenciados pela imersão aquática destaca-se a freqüência cardíaca (FC), muito utilizada durante o exercício devido a sua simplicidade de medição e suas relações com o consumo de oxigênio e com a intensidade de trabalho (31). Sendo a FC utilizada para classificar a intensidade do esforço na elaboração de programas de treinamento (38) e diante das influências provocadas pelo meio aquático, a aplicação da FC adequada a um treinamento em terra nos programas de exercícios físicos aquáticos é questionável. Para Sheldahl (33), as alterações na FC em ambiente aquático são causadas pela temperatura da água e/ou pela hipervolemia central, e a FC para o treinamento na água não deve ser a mesma dos exercícios em terra. O comportamento da FC durante a imersão tem relação com a manutenção da temperatura corporal. Uma das principais respostas fisiológicas decorrentes da exposição generalizada ao frio é a vasoconstrição periférica, que desvia o sangue da superfície da pele para áreas centrais; portanto, a diminuição da temperatura deve causar redução na FC (4). Um corpo imerso na água participa de um sistema dinâmico, havendo troca de energia calórica entre este corpo e a água até equilibrar o sistema. Um volume de água retira mais calor que um volume igual de ar e esta particularidade faz com que o organismo remova mais calor quando em água fresca (33). Segundo Kollias et al. (18), a perda de calor na água é considerada cerca de 25 vezes maior em comparação com o ar. Esta perda de calor durante o exercício na água é diferente da mesma situação em terra, visto que a evaporação, que é a forma principal de dissipação de calor em terra, não ocorre na água. Em contrapartida, a perda ou o ganho através da convecção e condução é muito maior no meio aquático que no meio terrestre (11).

Pesquisas relativas ao comportamento da FC em situação de imersão aquática já encontraram tanto taquicardia $(7,16,17,39)$ quanto nenhuma alteração na FC $(1,3)$ e, contudo, a maior parte dos estudos aponta para bradicardia $(6,8,10,14,15,19,20$, $21,23,24,29,30,31,32,36,37,38)$. Ao acrescentar a variável temperatura do meio aquático, as opiniões dividem-se entre bradicardia $(5,13,25,26,27$, $28,34,35)$ ou taquicardia $(34,35)$ aumentadas com a redução da temperatura, e também manutenção da FC mesmo com as alterações na temperatura $(4,11$, 28), embora a tendência mais forte seja aceitar o aumento da bradicardia causado pela diminuição da temperatura da água.

$\mathrm{Na}$ tentativa de elucidar melhor o comportamento da FC durante a imersão em condições de profundidade, posicionamento corporal e variação térmica que correspondam às condições mais comumente utilizadas para a prática de hidroginástica, o objetivo deste estudo foi analisar o comportamento da FC em indivíduos imersos na posição vertical, na profundidade entre apêndice xifóide e ombros, nas temperaturas aquáticas de 27,30 e $33^{\circ} \mathrm{C}$.

\section{MATERIAL E MÉTODOS}

A amostra do presente estudo foi composta por 14 indivíduos do sexo masculino, praticantes de atividades aquáticas há, no mínimo, um ano, visando evitar que a não familiarização com o meio aquático pudesse influenciar no comportamento da FC. Todos os indivíduos eram isentos de doenças e com idades entre 18 e 35 anos.

As informações sobre o protocolo de coleta de dados foram fornecidas por escrito aos integrantes da amostra, juntamente com o convite para a participação no estudo e o termo de consentimento, o qual foi devida- 
mente assinado por todos. Este estudo foi aprovado pelo Comitê de Ética em Pesquisa em Seres Humanos da Universidade do Estado de Santa Catarina. Para verificar a FC, utilizou-se um sensor de batimentos cardíacos da marca Polar, modelo Beat. Para a verificação da temperatura da água, foi utilizado um termômetro químico, de líquido vermelho, da marca Incoterm, apresentando resolução de $0,5^{\circ} \mathrm{C}$. Já para a medida da temperatura e da umidade do ambiente da piscina, utilizou-se um termo -higrômetro de bulbo seco e úmido, da marca Incoterm, apresentando resolução de $1^{\circ} \mathrm{C}$. A temperatura e a umidade relativa do ambiente foram registradas a cada inter valo de uma hora.

Os dados foram coletados em piscina localizada em ambiente fechado, com 1,20 $\mathrm{m}$ de profundidade, permitindo que a profundidade de imersão ficasse entre o apêndice xifóide e os ombros dos sujeitos. A coleta dos dados ocorreu em 3 etapas, com diferentes temperaturas aquáticas $\left(1^{\text {a }}\right.$ etapa: $33^{\circ} \mathrm{C}, 2^{\text {a }}$ etapa: $30^{\circ} \mathrm{C}$, $3^{\text {a }}$ etapa: $27^{\circ} \mathrm{C}$ ) e inter valo de 7 dias entre cada etapa. O horário de coleta dos dados foi o mesmo nos diferentes dias, abrangendo o período da tarde. Durante todo o período de coleta, os indivíduos mantiveram suas rotinas e hábitos de vida inalterados.

Inicialmente, foram realizadas as medidas antropométricas dos indivíduos da amostra. Os indivíduos foram equipados com o sensor de batimentos cardíacos e receberam novamente as informações sobre os procedimentos aos quais seriam submetidos. A seguir, foi verificada a FC de repouso, obtida após a permanência em repouso por 10 minutos, na posição deitado, ao lado da piscina. Logo após, imediatamente antes de entrar calmamente na piscina, foi registrada a FC inicial, na posição de pé. Então, cada indivíduo entrou na piscina e colocou-se na posição básica: descontraidamente em pé, braços relaxados, joelhos flexionados, permitindo atingir a profundida- de de imersão entre o apêndice xifóide e os ombros. Decorridos 2 minutos, foi coletada a FC final. A temperatura ambiente durante a coleta dos dados permaneceu entre $21,5^{\circ} \mathrm{C}$ e $23^{\circ} \mathrm{C}$.

O comportamento da FC foi analisado através das variações existentes entre a FC inicial e a FC final, nas 3 diferentes condições de temperatura da água (33, 30 e $\left.27^{\circ} \mathrm{C}\right)$.

Para a análise dos dados coletados, foi utilizada a estatística descritiva, o teste de normalidade de Shapiro -Wilks, o teste de homogeneidade de Levene, análise de variância (ANOVA) e teste F para comparar as classes das variáveis classificatórias. Para a localização das diferenças, utilizou-se o teste post-hoc de Bonferroni $(\mathrm{p} \leq 0,05)$. Foi utilizado o pacote estatístico computacional SPSS for Windows, versão 8.0.

\section{RESULTADOS}

A caracterização da amostra é apresentada na tabela 1 .

Tabela 1. Médias e desvios-padrão (DP) da idade, tempo de prática de atividades aquáticas, massa, estatura e índice de massa corporal.

\begin{tabular}{lcc}
\hline VARIÁVEL & MÉDIA & DP \\
\hline Idade (anos) & 25,21 & $\pm 5,81$ \\
Tempo de prática de atividades & 9,71 & $\pm 5,98$ \\
aquáticas (anos) & & \\
Massa $(\mathrm{kg}$ ) & 75,99 & $\pm 7,21$ \\
Estatura (m) & 1,76 & $\pm 0,05$ \\
Índice de massa corporal $\left[\mathrm{kg} \cdot \mathrm{m}^{-2}\right.$ ) & 24,39 & $\pm 1,48$ \\
\hline
\end{tabular}

Os resultados nas diferentes etapas da coleta dos dados demonstraram uma distribuição normal e homogênea. As médias e desvios-padrão dos valores da FC de repouso e da FC inicial, na situação fora da água, obtidos nas diferentes etapas são apresentados na tabela 2. Não foram encontradas diferenças estatisticamente significativas para os valores da FC de repouso e da FC inicial entre as 3 diferentes etapas.

Tabela 2. Médias e desvios-padrão (DP) da FC de repouso e da FC inicial nas diferentes etapas da coleta dos dados e resultados da análise de variância.

\begin{tabular}{|c|c|c|c|c|c|c|c|}
\hline \multirow[t]{2}{*}{$\mathrm{FC}$} & \multicolumn{2}{|c|}{$1^{a}$ etapa } & \multicolumn{2}{|c|}{$2^{a}$ etapa } & \multicolumn{2}{|c|}{$3^{2}$ etapa } & \multirow[t]{2}{*}{ SIG. } \\
\hline & $\begin{array}{l}\text { MÉDIA } \\
\text { (bpm) }\end{array}$ & $\begin{array}{c}\text { DP } \\
\text { (bpm] }\end{array}$ & $\begin{array}{l}\text { MÉDIA } \\
\text { (bpm) }\end{array}$ & $\begin{array}{c}\mathrm{DP} \\
\text { (bpm) }\end{array}$ & $\begin{array}{l}\text { MÉDIA } \\
\text { (bpm) }\end{array}$ & $\begin{array}{c}\text { DP } \\
\text { (bpm) }\end{array}$ & \\
\hline Repouso & 66,23 & $\pm 8,69$ & 66,00 & $\pm 9,89$ & 64,46 & $\pm 5,97$ & 0,833 \\
\hline Inicial & 91,54 & $\pm 11,90$ & 91,57 & $\pm 13,56$ & 96,00 & $\pm 9,83$ & 0,557 \\
\hline
\end{tabular}


As médias, desvios-padrão, limites inferiores e superiores do inter valo de confiança, e os valores mínimos e máximos da bradicardia encontrados durante a imersão nas diferentes temperaturas aquáticas são apresentados na tabela 3. Esses dados demonstram uma redução média na FC, comparada com a posição inicial fora da água, que varia de 17,85 $\pm 10,67 \mathrm{bpm}$ na temperatura aquática de $33^{\circ} \mathrm{C}$ a $33,75 \pm 11,27$ bpm na temperatura aquática de $27^{\circ} \mathrm{C}$. Constata-se, ainda, uma redução média não significativa de 7 bpm da temperatura de $33^{\circ} \mathrm{C}$ para $30^{\circ} \mathrm{C}(\mathrm{p}=0,44)$ e de $9 \mathrm{bpm}$ da temperatura de $30^{\circ} \mathrm{C}$ para $27^{\circ} \mathrm{C}$ $(p=0,10)$. No entanto, ressalta-se a redução estatisticamente significativa de $16 \mathrm{bpm}$ na $\mathrm{FC}(\mathrm{p}=0,003)$ da temperatura de $33^{\circ} \mathrm{C}$ para $27^{\circ} \mathrm{C}$.

Foi encontrada, portanto, redução na FC durante a imersão em todos os indivíduos analisados, nas três diferentes temperaturas aquáticas utilizadas para coletar os dados, com uma bradicardia mais acentuada conforme a diminuição da temperatura da água.

Tabela 3. Médias, desvios-padrão (DP], valores mínimos e máximos da bradicardia nas diferentes temperaturas aquáticas.

\begin{tabular}{|c|c|c|c|c|c|c|}
\hline \multirow[t]{2}{*}{$\begin{array}{l}\text { TEMP } \\
{\left[{ }^{\circ} \mathrm{C}\right]}\end{array}$} & \multirow[t]{2}{*}{$\begin{array}{l}\text { MÉDIA } \\
\text { [bpm] }\end{array}$} & \multirow[t]{2}{*}{$\begin{array}{c}\mathrm{DP} \\
{[\mathrm{bpm}]}\end{array}$} & \multicolumn{2}{|c|}{$\begin{array}{l}\text { 95\% DO INTERVALO DE } \\
\text { CONFIANÇA DA MÉDIA }\end{array}$} & \multirow[t]{2}{*}{$\begin{array}{l}\text { MÍNIMO } \\
\text { [bpm] }\end{array}$} & \multirow[t]{2}{*}{$\begin{array}{l}\text { MÁXIMC } \\
\text { [bpm] }\end{array}$} \\
\hline & & & $\begin{array}{l}\text { LIMITE INFERIOR } \\
\text { (bpm) }\end{array}$ & $\begin{array}{l}\text { LIMITE SUPERIOR } \\
\text { (bpm) }\end{array}$ & & \\
\hline 33 & $17,85^{3}$ & $\pm 10,6 ?$ & 11,40 & 24,30 & 5,0 & 43,0 \\
\hline 30 & $24,14^{\mathrm{ab}}$ & $\pm 11,16$ & 17,70 & 30,59 & 2,0 & 48,0 \\
\hline 27 & $33,75^{b}$ & $\pm 11,27$ & 26,60 & 40,90 & 17,0 & 56,0 \\
\hline
\end{tabular}

Nota: letras diferentes indicam diferenças estatisticamente significativas para $p<0,05$.

\section{DISCUSSÃO}

O comportamento semelhante dos valores médios da FC de repouso e da FC inicial, entre as 3 diferentes etapas de coleta dos dados, indica que a magnitude das alterações encontradas na FC, durante a imersão aquática, foi provocada pelas diferentes temperaturas de água utilizadas.

A bradicardia durante a imersão encontrada no presente estudo, independente da temperatura aquática, corrobora com os dados de diversas pesquisas $(6,8$, $10,14,15,19,20,21,23,24,29,30,31,32,36,37$, 38). Na maioria dos estudos citados, a amostra foi composta por indivíduos na faixa etária dos 17 aos 35 anos, praticantes de atividades físicas regulares. Além disso, a faixa de variação da temperatura aquática utilizada situou-se entre 25 e $35^{\circ} \mathrm{C}$, temperaturas inferiores à termoneutra em relação à $\mathrm{FC}$ para repouso. Alguns estudos avaliaram a FC durante o repouso $(19,21,29,30)$, os demais durante o exercício físico, submáximo ou máximo. A profundidade e a posição de imersão adotadas também variaram dentre os estudos, sendo fornecidos dados que apresentaram reduções maiores ou menores na FC, de acordo com a situação específica.

Com resultados discordantes dos expostos acima, outros estudos $(1,3,7,12,16,17,22,39)$ não encontraram redução na FC durante a imersão aquática. Tais resultados podem ser justificados ao serem analisados alguns aspectos metodológicos. Um dos aspectos consiste nas temperaturas aquáticas utilizadas, pois a temperatura termoneutra não provoca diferenças no comportamento da FC $(1,3,7)$ e temperaturas superiores àquela considerada termoneutra provocam aumento na FC (17). Outro aspecto envolve tipos e intensidades de exercício, bem como os indicadores de intensidade do esforço adotados como controle. Quando movimentos similares são executados com igual velocidade nos meios aquático e terrestre, as intensidades do esforço não são necessariamente idênticas, fazendo com que a caminhada aquática $(22,39)$ ou determinados exercícios aquáticos localizados (16) correspondam a uma maior sobrecarga e, conseqüentemente, exijam uma maior FC. 
A influência da temperatura da água é considerada com maior relevância nas pesquisas realizadas por Craig e Dvorak (5), Costill et al. (4), Rennie et al. (28), Holmér e Bergh (13), McArdle et al. (25), McMurray e Horvath (26), Shimizu et al. (34), Park et al. (27), Srámek et al. (35) e Fujishima et al. (11), as quais utilizaram duas ou mais temperaturas para coletar os dados. Podem ser consideradas semelhantes ao presente estudo, visto que objetivaram comparar as respostas da $\mathrm{FC}$ às diferentes temperaturas de imersão aquática. As amostras das pesquisas selecionadas envolveram indivíduos jovens e adultos, fisicamente ativos, com idades entre 14 e 34 anos. As temperaturas aquáticas utilizadas para coletar os dados variaram entre 14 e $37^{\circ} \mathrm{C}$, permitindo uma visão abrangente sobre o comportamento da FC nestas diferentes temperaturas.

Dos estudos citados, apenas Costill et al. (4) e Fujishima et al. (11) não encontraram diferenças significativas no comportamento da FC em diferentes temperaturas de água. Ambos os estudos analisaram as respostas fisiológicas durante o exercício de natação, que é realizado na posição supina. Costill et al. (4) não relataram diferenças significativas na FC durante 20 minutos de natação, em intensidade submáxima constante, em temperaturas de água de $17,4,26,8$ e $33,1^{\circ} \mathrm{C}$. No estudo de Fujishima et al. (11), foi obser vada uma FC significativamente mais alta para o exercício em esteira, realizado fora da água, comparado à natação, com todos os testes realizados em uma mesma intensidade de esforço. Da mesma forma que no estudo anterior, não foram relatadas diferenças significativas durante o exercício em imersão em temperaturas de 23,28 e $33^{\circ} \mathrm{C}$.

Assim, a semelhança no comportamento da FC entre as diferentes temperaturas utilizadas não está bem esclarecida pelos autores, fato este que pode ser atribuído à metodologia utilizada.

No presente estudo, realizado em repouso e em imersão vertical, a diminuição da temperatura aquática de $33^{\circ} \mathrm{C}$ para $27^{\circ} \mathrm{C}$ provocou redução média de 16 bpm na FC, corroborando com outros estudos que já haviam relatado a ocorrência de redução nos batimentos cardíacos ao ser diminuída a temperatura da água $(13,25,26)$. Os estudos citados, entretanto, avaliaram o comportamento da FC durante o esfor- ço, revelando diferenças nos mecanismos de perda de calor e, conseqüentemente, na magnitude das alterações na FC. Holmér e Bergh (13) relataram redução de $7 \mathrm{bpm}$, ao comparar a FC obtida na temperatura aquática de $34^{\circ} \mathrm{C}$ com aquela obtida na temperatura de $26^{\circ} \mathrm{C}$, e de $15 \mathrm{bpm}$ dos $34^{\circ} \mathrm{C}$ para os $18^{\circ} \mathrm{C}$, durante a natação em swimming flume.

Resultados semelhantes foram apontados por McArdle et al. (25), que encontraram diminuição de $10 \mathrm{bpm}$ da temperatura de $33^{\circ} \mathrm{C}$ para $25^{\circ} \mathrm{C}$ e de 15 bpm dos $33^{\circ} \mathrm{C}$ para os $18^{\circ} \mathrm{C}$, durante exercício em cicloergômetro aquático. Também utilizando cicloergômetro aquático, McMurray e Horvath (26) verificaram redução de $6 \mathrm{bpm}$ da temperatura de $35^{\circ} \mathrm{C}$ para a temperatura de $30^{\circ} \mathrm{C}$, de $17 \mathrm{bpm}$ dos $35^{\circ} \mathrm{C}$ para os $25^{\circ} \mathrm{C}$ e de $19 \mathrm{bpm}$ dos $35^{\circ} \mathrm{C}$ para os $20^{\circ} \mathrm{C}$. Assim, as diferenças na magnitude da redução dos batimentos cardíacos que acompanham a diminuição na temperatura da água, ao comparar os estudos citados com o presente estudo, podem ser devidas à atividade física. Os dados apresentados indicam que tal redução na FC é menos pronunciada durante o exercício, visto que o calor gerado pelo trabalho muscular auxilia a equilibrar a perda de calor (13). Em pesquisas em condição de repouso, Park et al. (27) encontraram valores semelhantes entre o meio terrestre e imersão aquática em $34,5^{\circ} \mathrm{C}$, mas encontraram uma bradicardia significativa de $11 \mathrm{bpm}$ em imersão na temperatura de $30^{\circ} \mathrm{C}$. Já Srámek et al. (35) encontraram uma bradicardia de $9 \mathrm{bpm}$ em imersão em $32^{\circ} \mathrm{C}$ e $20^{\circ} \mathrm{C}$, enquanto que em imersão na temperatura de $14^{\circ} \mathrm{C}$, encontraram um aumento na FC de $3 \mathrm{bpm}$. Esse aumento pode ser explicado pelos tremores obser vados, que podem ser considerados como um exercício leve. Segundo esses autores, a imersão em temperaturas próximas à termoneutra conduz a uma bradicardia, visto que ativa diferentes sistemas regulatórios e diferentes mecanismos efetores, comparativamente à imersão em água fria, como na temperatura de $14^{\circ} \mathrm{C}$, que estimula a um aumento na FC.

Rennie et al. (28) não especificaram os valores de FC encontrados no seu estudo, apenas afirmaram não ocorrer bradicardia na temperatura aquática de $36^{\circ} \mathrm{C}$, e apresentaram um percentual de 20 a $25 \%$ para a bradicardia existente em temperaturas aquá- 
ticas abaixo de $34^{\circ} \mathrm{C}$. Craig e Dvorak (5) também não fornecem os valores da FC coletada na referida pesquisa, contudo afirmam haver um aumento na FC em temperaturas aquáticas de 36 e $37^{\circ} \mathrm{C}$ e uma redução na $\mathrm{FC}$ em temperaturas abaixo dos $35^{\circ} \mathrm{C}$. Shimizu et al. (34) também encontraram uma FC mais elevada em exercício, para uma mesma taxa de $\mathrm{VO}_{2}$, em temperatura de $35^{\circ} \mathrm{C}$, com bradicardia significativa nas temperaturas de 25 e $30^{\circ} \mathrm{C}$.

Entretanto, a FC na temperatura de $25^{\circ} \mathrm{C}$ foi significativamente maior que a obser vada em $30^{\circ} \mathrm{C}$. As demandas termorregulatórias podem ser excluídas como explicação para uma FC mais elevada na temperatura de $25^{\circ} \mathrm{C}$, pois se, por um lado, a demanda de fluxo sangüíneo para a pele foi mais baixa, por outro, os níveis de $\mathrm{VO}_{2}$ foram semelhantes para as três temperaturas utilizadas, excluindo a possibilidade de excesso de produção de calor na água fria. Assim, a mais alta $\mathrm{FC}$ na temperatura de $35^{\circ} \mathrm{C}$, comparada a $30^{\circ} \mathrm{C}$, pode ser explicada por um aumento na ativação simpática do coração, devido às demandas de fluxo sangüíneo para pele. Já a maior $\mathrm{FC}$ em $25^{\circ} \mathrm{C}$ pode representar uma ativação refle xa do sistema nervoso simpático pelos receptores de frio na pele.

Segundo McArdle et al. (25), há redução na circulação sangüínea periférica com a imersão em água fria, como forma de impedir a dissipação do calor corporal e manter o equilíbrio térmico do organismo. A ação conjunta da pressão hidrostática e da vasoconstrição periférica seria responsável por aumentos no volume sangüíneo central e no retorno venoso, resultando em maior volume de ejeção sistólica para justificar a bradicardia existente.

McArdle et al. (25) afirmam que a bradicardia relatada durante a imersão não é um efeito inerente à própria imersão, mas uma resposta à temperatura da água. Este fato foi comprovado por Rennie et al. (28) e Craig e Dvorak (5), pois seus estudos demonstram que a FC permanece igual ou mostra aumento em temperaturas aquáticas de 36 ou $37^{\circ} \mathrm{C}$, enquanto ocorre bradicardia abaixo de 35 ou $34^{\circ} \mathrm{C}$. A temperatura termoneutra em relação à $\mathrm{FC}$, ou seja, aquela temperatura que não provoca desequilíbrio térmico corporal causador de alterações na FC, situa-se entre 35 e $35,5^{\circ} \mathrm{C}$ (5). Svedenhag e Seger
(36) confirmam que os ajustes cardiovasculares durante a imersão dependem da temperatura da água, reforçando o quanto a vasoconstrição e a vasodilatação são importantes na hemodinâmica. Dessa forma, pode-se inferir que, com a diminuição da temperatura da água, o maior stress provocado pelo frio seria responsável pelo aumento da bradicardia. Vale relembrar que a termocondutividade da água é cerca de 25 vezes maior que a do ar (18), produzindo maior perda de calor. Os ajustes a temperaturas inferiores incluem vasoconstrição periférica e bradicardia elevada, como medidas preventivas da hipotermia.

\section{CONCLUSÃO}

Os dados obtidos neste estudo permitem concluir que a temperatura da água exerce efeito sobre a FC de indivíduos adultos do sexo masculino, fisicamente ativos e isentos de doenças, submetidos à imersão na posição vertical, na profundidade entre apêndice xifóide e ombros. A variação existente na FC durante a imersão vertical aquática nas temperaturas de 33,30 e $27^{\circ} \mathrm{C}$ consiste em diminuição dos batimentos cardíacos por unidade de tempo. Ainda, as alterações significativas na FC decorrentes das modificações nas condições térmicas de imersão indicam aumento da bradicardia quando a temperatura aquática é diminuída, considerando -se a faixa de variação entre 33 e $27^{\circ} \mathrm{C}$.

Aceita-se que as alterações na FC encontradas consistam em indicadores dos mecanismos fisiológicos responsáveis pela manutenção da temperatura corporal, diretamente influenciada pela temperatura do ambiente. Este conhecimento deve ser aplicado na prescrição e no controle da intensidade dos exercícios aquáticos, visto que não é suficiente saber quais são as variações apresentadas pela FC durante a imersão, mas também se estas são provocadas pela temperatura da água ou pela intensidade do exercício. Em outras palavras, ao prescrever a intensidade do esforço por meio da FC, deve-se considerar, entre outros fatores como a posição do corpo e sua profundidade de imersão, a temperatura na qual o exercício será realizado. 


\section{AGRADECIMENTOS}

Agradecemos aos proprietários do Centro Ortopédico, São Leopoldo - Rio Grande do Sul, por terem gentilmente cedido suas instalações para a realização da coleta de dados do presente estudo.

\section{CORRESPONDÊNCIA}

Fabiane Graef

Grupo de Pesquisa em Atividades Aquáticas Laboratório de Pesquisa do Exercício

Escola de Educação Física

Universidade Federal do Rio Grande do Sul Rua Felizardo, 750 - Bairro Jardim Botânico 90690-200 - Porto Alegre/RS.

fgmuller@terra.com.br 


\section{REFERÊNCIAS BIBLIOGRÁFICAS}

1. Arborelius M, Balldin UI, Lilja B, Lundgren, CEG (1972). Hemodynamic changes in man during immersion with the head above water. Aerospace Medicine 43(6): 592-598.

2. Avellini BA, Shapiro Y, Pandolf KB (1983). Cardio -respiratory physical training in water and on land. Eur J Appl Physiol 50: 255-263.

3. Begin R, Epstein M, Sackner MA, Levinson R, Dougherty R, Duncan D (1976). Effects of water immersion to the neck on pulmonar y circulation and tissue volume in man. J Appl Physiol 40(3): 293-299.

4. Costill DL, Cahill PJ, Eddy D (1967). Metabolic responses to submaximal exercise in three water temperatures. $J$ Appl Physiol 22(4): 628-632.

5. Craig AB, Dvorak $M$ (1966). Thermal regulation during water immersion. J Appl Physiol 21(5): 1577-1585.

6. Denadai BS, Rosas R, Denadai MLDR (1997). Limiar aeróbio e anaeróbio na corrida aquática: comparação com os valores obtidos na corrida em pista. Revista Brasileira de Atividade Física e Saúde 2(1): 23-28.

7. Denison DM, Wagner PD, Kingaby GL, West JB (1972). Cardiorespirator y responses to exercise in air and underwater. J Appl Physiol 33(4): 426-430.

8. Dixon RW, Faulkner JA (1971). Cardiac outputs during maximum effort running and swimming. J Appl Physiol 30(5): 653-656.

9. Farhi LE, Linnarsson D (1977). Cardiopulmonar y readjustments during graded immersion in water at $35^{\circ} \mathrm{C}$. Respir Physiol 30: 35-50.

10. Frangolias DD, Rhodes EC (1995). Maximal and ventilatory threshold responses to treadmill and water immersion running. Med Sci Sports Exerc 27(7): 1007-1013.

11. Fujishima K, Shimizu T, Ogaki T, Hotta N, Kanaya S, Shono R, Ueda T (2001). Thermoregulator y responses to low-intensity prolonged swimming in water at various temperatures and treadmill walking on land. J Physiol Anthropol 20(3): 199-206.

12. Green JH, Cable NT, Elms N (1990). Heart rate and oxygen consumption during walking on land and in deep water. J Sports Med Phys Fitness 30: 49-52.

13. Holmér I, Bergh U (1974). Metabolic and thermal response to swimming in water at varying temperatures. $J A p p l$ Physiol 37(5): 702-705.

14. Holmér I, Lundin A, Eriksson BO (1974a). Maximum oxygen uptake during swimming and running by elite swimmers. J Appl Physiol 36(6): 711-714.

15. Holmér I, Stein EM, Saltin B Ekblom B, Astrand PO (1974b). Hemodynamic and respirator y responses compared in swimming and running. J Appl Physiol 37(1): 49-54.

16. Johnson BL, Stramlne SB, Adamezyk JW (1977).

Comparison of oxygen uptake and heart rate during exerci ses on land and in water. Phys Ther 57: 273-278.

17. Kenny GP, Giesbrecht GG, Thoden JS (1996). A comparison of human thermoregulator y response following dynamic exercise and warm-water immersion. Eur J Appl Physiol 74: 336-341.

18. Kollias J, Barlett L, Bergsteinova V, Skinner JS, Buskirk ER, Nicholas WC (1974). Metabolic and thermal responses of women during cooling in water. J Appl Physiol 36(5): 577580

19. Kruel LFM (1994). Peso hidrostático e freqüência cardíaca em pessoas submetidas a diferentes profundidades de água. Dissertação de Mestrado. Centro de Educação Física e Desportes, Universidade Federal de Santa Maria, Brasil.
20. Kruel LFM, Moraes EZC, Ávila AOV, Sampedro RMF (2001). Alterações fisiológicas e biomecânicas em indivíduos praticando exercícios de hidroginástica dentro e fora d'água. Revista Kinesis no especial: 104-129.

21. Kruel LFM, Tartaruga LAP, Dias AC, Silva RC, Picanço PSP, Rangel AB (2002). Freqüência cardíaca durante imersão no meio aquático. Fitness e Performance 1(6): 46-51.

22. Lazzari JMA, Meyer F (1997). Freqüência cardíaca e percepção do esforço na caminhada aquática e na esteira em mulheres sedentárias e com diferentes percentuais de gordura. Revista Brasileira de Atividade Física e Saúde 2(3): 7-13.

23. Magel JR, Faulkner JÁ (1967). Maximum oxygen uptak es of college swimmers. J Appl Physiol 22(5): 929-938.

24. McArdle WD, Glaser RM, Magel JR (1971). Metabolic and cardiorespirator y response during free swimming and treadmill walking. J Appl Physiol 30(5): 733-738.

25. McArdle WD, Magel JR, Lesmes GR, Pechar GS (1976). Metabolic and cardiovascular adjustment to work in air and water at 18,25 and $33^{\circ} \mathrm{C}$. J Appl Physiol 40(1): 85-90

26. McMurray RG, Horvath SM (1979). Thermoregulation in swimmers and runners. J Appl Physiol 46(6): 1086-1092.

27. Park KS, Choi JK, Park YS (1999). Cardiovascular regulation during water immersion. Appl Human Sci 18(6): 233 241.

28. Rennie DW, Di Prampero P, Cerretelli P (1971). Effects of water immersion on cardiac output, heart hate and stroke volume of man at rest and during exercise. Medicina dello Sport 24: 223-228.

29. Risch WD, Koubenec HJ, Beckmann U, Lange S, Gauer OH. (1978a). The effect of graded immersion on heart volume, central venous pressure, pulmonar y blood distribution, and heart rate in man. Pflügers Archiv 374: 115118.

30. Risch WD, Koubenec HJ, Gauer OH, Lange S (1978b) . Time course of cardiac distension with rapid immersion in a thermo -neutral bath. Pflügers Archiv 374: 119-120.

31. Scolfaro LB, Marins JCB, Regazzi AJ (1988). Estudo comparativo da freqüência cardíaca máxima em três modalidades cíclicas. Revista da APEF 13(1): 44-54.

32. Sheldahl LM, Wann LS, Clifford PS, Tristani FE, Wolf LG Kalbeleish JH (1984). Effect of central hyper volemia on cardiac performance during exercise. J Appl Physiol 52: 1662-1667.

33. Sheldahl LM (1985). Special ergometric techniques and weight reduction. Med Sci Sports Exerc 18(1): 25-30.

34. Shimizu T, Kosak a M, Fujishima K (1998). Human thermoregulator y responses during prolonged walking in water at 25, 30 and $35^{\circ} \mathrm{C}$. J Appl Physiol 78: 473-478.

35. Srámek P, Simeck ová M, Janski L, Savlík ová J, Vybíral S (2000). Human Physiological responses to immersion into water of different temperatures. Eur J Appl Physiol 81: 436442 .

36. Svedenhag J, Seger J (1992). Running on land and in water: comparative exercise physiology. Med Sci Sports Exerc 24(10): 1155-1160.

37. Town GP, Bradley SS (1991). Maximal metabolic responses of deep and shallow water running in trained runners. Med Sci Sports Exerc 23(2): 238-241.

38. Vilas-Boas JP (1989). Valores máximos da freqüência cardíaca obtidos em natação e em tapete rolante. Revista Portuguesa de Medicina Desportiva 7: 109-125.

39. Whitley JD, Schoene LL (1987). Comparison of heart rate responses: water walking versus treadmill walking. Phys Ther 10: 1501-1504. 